\title{
Computational Study of Induction Heating Process in Crystal Growth Systems-The Role of Input Current Shape
}

\author{
Mohammad Hossein Tavakoli ${ }^{1 *}$, Tayebe Nadery Mostagir ${ }^{2}$ \\ ${ }^{1}$ Physics Department, Bu-Ali Sina University, Hamedan, Iran \\ ${ }^{2}$ Department of Physics, Kurdistan University, Sanandaj, Iran \\ Email: ${ }^{*}$ mht@basu.ac.ir, mhtvkl@gmail.com
}

Received October 28, 2012; revised November 30, 2012; accepted December 8, 2012

\begin{abstract}
A set of 2D steady state finite element numerical simulations of electromagnetic fields and heating distribution for an oxide Czochralski crystal growth system was carried out for different input current shapes (sine, square, triangle and sawtooth waveforms) of the induction coil. Comparison between the results presented here demonstrates the importance of input current shape on the electromagnetic field distribution, coil efficiency, and intensity and structure of generated power in the growth setup.
\end{abstract}

Keywords: Computer Simulation; Induction Heating; Czochralski Method; Growth from Melt; Metals

\section{Introduction}

Radio frequency induction heating is frequently used in crystal growth technology. The process principle consists of applying an alternating current in a conductor or coil called inductor (RF-coil) that generates an alternating electromagnetic field in the space. The alternating electromagnetic field induces eddy currents in metal crucible where the crystal material is placed and should be to melt. These currents lead to Joulean heating $\left(R I^{2}\right)$ of the crucible in the form of temporal and spatial volumetric heating. Distribution and control of the induced power along the crucible cross-section and length are quite important which result in temperature difference and flow field in the growth setup [1-3].

In order to produce the required heating pattern within a metal crucible and afterheater it is necessary to accurately model and predict the electromagnetic field and the eddy currents distribution produced by the RF-coil under different operating conditions such as geometry and orientation of the metallic parts, cross section of the coil turns, the crucible shape and position, and frequency choice [4-7]. Selection of input current shape is another critical issue, which is particularly important for certain selective heating applications. In this article, we try to investigate the effects of different input voltage shapes, i.e., sine, square, triangle and sawtooth waveforms (Fig-

${ }^{*}$ Corresponding author. ure 1) on the strength and distribution of the electromagnetic fields and heat generation in a Czochralski setup using the mathematical modeling and computer simulation. It should be noted, however, that despite of the differences in the patterns, each pattern is periodic. This point is important for our analysis of the driving current shape, i.e., they can be represented as closely as desired by the combination of a sufficiently large number of sinusoidal patterns that form a harmonic series (Fourier series). Every non-sinusoidal current pattern consists of a fundamental and a complement of harmonics, which can be considered as a superposition of sine pattern of a fundamental frequency $\omega$ and integer multiples of that frequency [8].

\section{Mathematical Model}

\subsection{Governing Equations}

Since the real induction heating process is very complex, we make some simplifying assumptions in our approach. In our mathematical model used for numerical calculations, we make the following five assumptions: 1) the heating system is rotationally symmetric about the z-axis, so that all quantities are independent of the azimuthal coordinate $\varphi ; 2$ ) all materials are isotropic, non-magnetic and have no net electric charge; 3 ) the displacement current is neglected; 4) the distribution of driving electrical current (also voltage) in the RF-coil is uniform; and 5) 

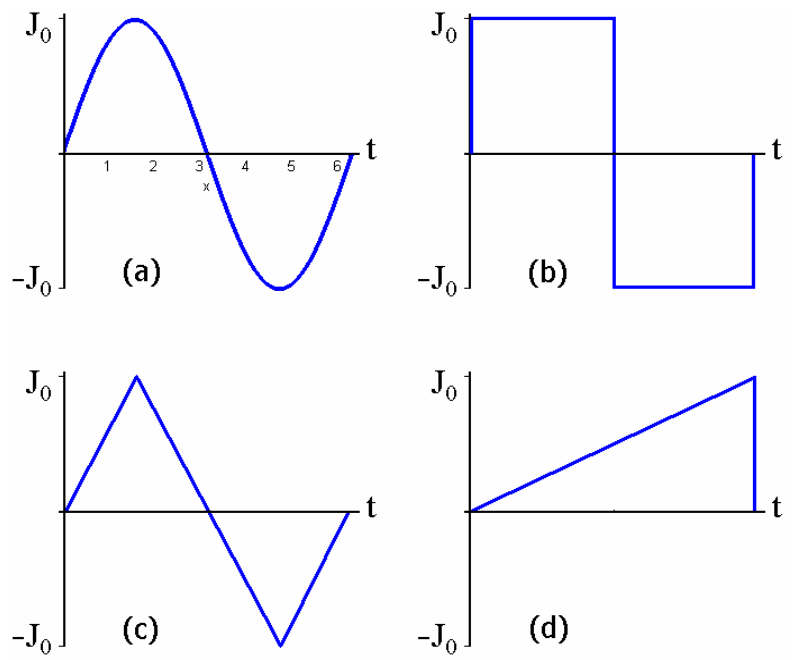

Figure 1. Four input current shapes (a) Sine; (b) Square; (c) Triangle; and (d) Sawtooth waveforms of the induction coil.

the driving and induced currents have only one angular component (i.e., $\varphi$-direction). Under these assumptions, the governing equations are [4];

$$
\frac{\partial}{\partial r}\left(\frac{1}{r} \frac{\partial \psi_{B}}{\partial r}\right)+\frac{\partial}{\partial z}\left(\frac{1}{r} \frac{\partial \psi_{B}}{\partial r}\right)=\hat{\mathcal{L}} \psi_{B}=-\mu_{0} J_{\varphi}
$$

where

$$
\hat{\mathcal{L}}=\frac{\partial}{\partial r}\left(\frac{1}{r} \frac{\partial \psi_{B}}{\partial r}\right)+\frac{\partial}{\partial z}\left(\frac{1}{r} \frac{\partial \psi_{B}}{\partial r}\right)
$$

and

$$
J_{\varphi}=\left\{\begin{aligned}
& J_{d}^{\text {coil }}+J_{e}^{\text {coil }}= J_{d}^{\text {coil }}-\frac{\sigma_{c o}}{r} \frac{1}{r} \frac{\partial \psi_{B}}{\partial t} \\
& \text { driving and eddy currents in the coil } \\
& J_{e}^{\text {crucible }}=-\frac{\sigma_{c r}}{r} \frac{1}{r} \frac{\partial \psi_{B}}{\partial t} \text { eddy currents in the crucible }
\end{aligned}\right.
$$

in which $\psi_{B}$ is the magnetic stream function defined by $\psi_{B}(r, z, t) \equiv r A_{\varphi}(r, z, t)$, where $A_{\varphi}$ is the azimuthal component of the vector potential, $(r, z)$ the cylindrical coordinates, $J$ the charge current density, $\sigma$ the electrical conductivity, $\mu_{0}$ the magnetic permeability of free space and $t$ the time.

The energy dissipation rate in all metallic parts (coil, crucible and afterheater) is computed as

$$
P(r, z, t)=\frac{J_{\varphi}^{2}}{\sigma}
$$

Finally we average over one period to obtain the volumetric heat generation rate (i.e., the time averaged quantity),

$$
q(r, z)=\frac{\omega}{2 \pi} \int_{0}^{\frac{2 \pi}{\omega}} \mathrm{P}(\mathrm{r}, \mathrm{z}, \mathrm{t}) \mathrm{dt}
$$

where $\omega$ is the frequency of the electrical current in the induction coil.

\section{a) Sine Waveform}

Assuming the driving current in the RF-coil as a sine form $J_{d}=J_{0} \sin \omega t$, we can consider a solution of the form

$$
\psi_{B}^{\operatorname{sine}}=S(r, z) \sin w t+C(r, z) \cos w t
$$

where $S(r, z)$ is the in-phase component and $C(r, z)$ is the out-of-phase component of the solution.

Now the coupled set of elliptic PDE's for $S(r, z)$ and $C(r, z)$ is:

$$
\begin{aligned}
& \hat{\mathcal{L}} S^{\text {sine }}=\left\{\begin{array}{l}
-\mu_{0}\left(J_{0}+\frac{\sigma_{c o} \omega}{r} C\right) \text { coil } \\
-\mu_{0} \frac{\sigma_{c o} \omega}{r} C \text { crucible } \\
0 \text { elsewhere }
\end{array}\right. \\
& \hat{\mathcal{L}} C^{\text {sine }}=\left\{\begin{array}{l}
\mu_{0} \frac{\sigma_{c o} \omega}{r} S \text { coil } \\
\mu_{0} \frac{\sigma_{c o} \omega}{r} S \text { crucible } \\
0 \text { elsewhere }
\end{array}\right.
\end{aligned}
$$

where $\hat{\mathcal{L}}$ is the linear operator defined in (2).

After solving (7) and (8) for $S(r, z)$ and $C(r, z)$, the eddy currents distribution and the energy dissipation rate can be computed via

$$
\begin{aligned}
J_{e}^{\text {sine }} & =-\frac{\sigma}{r} \frac{\partial \psi_{B}}{\partial t}=-\frac{\sigma \omega}{r}[S(r, z) \cos w t-C(r, z) \sin w t] \\
& =J_{C} \cos w t+J_{S} \sin w t
\end{aligned}
$$

and

$$
\begin{aligned}
& P(r, z, t) \\
= & \frac{J_{\varphi}^{2}}{\sigma} \\
= & \left\{\begin{array}{l}
\frac{\sigma_{c o} \omega^{2}}{r^{2}}\left[S^{2}+\left(\frac{J_{0 r}}{\sigma_{c o} \omega}+C\right)^{2}+S\left(\frac{J_{0 r}}{\sigma_{c o} \omega}+C\right)+\sin \omega t\right] \text { coil } \\
\frac{\sigma_{c r} \omega^{2}}{r^{2}}\left(S^{2}+C^{2}-C S \sin 2 \omega t\right) \text { crucible }
\end{array}\right.
\end{aligned}
$$

Consequently, the volumetric heat generation rate is

$$
q^{\text {sine }}(r, z)=\left\{\begin{array}{l}
\frac{\sigma_{c o} \omega^{2}}{r^{2}}\left[S^{2}+\left(\frac{J_{0 r}}{\sigma_{c o} \omega}+C\right)^{2}\right] \text { coil } \\
\frac{\sigma_{c r} \omega^{2}}{r^{2}}\left(S^{2}+C^{2}\right) \text { crucible }
\end{array}\right.
$$




\section{b) Square Waveform}

The square waveform of the driving current in the RFcoil can be approximated by a sum of harmonics using Fourier series as

$$
J_{d}=J_{0} F^{\text {square }}(t)=\frac{4 J_{0}}{\pi} \sum_{n=1}^{\infty} \frac{\sin (2 n-1) \omega t}{2 n-1}
$$

and then

$$
\begin{aligned}
& \psi_{B}^{\text {square }}=\sum_{n=1}^{\infty} \psi_{B(n)}^{\text {square }} \\
& =\sum_{n=1}^{\infty}\left[S_{n} \sin (2 n-1) \omega t+C_{n} \cos (2 n-1) \omega t\right] \\
& J_{e}^{\text {square }}=\sum_{n=1}^{\infty} J_{e(n)}^{\text {square }}=\sum_{n=1}^{\infty} \frac{(2 n-1) \sigma \omega}{r}\left[C_{n} \sin (2 n-1) \omega t\right. \\
& \left.-S_{n} \cos (2 n-1) \omega t\right] \\
& \hat{\mathcal{L}} S_{n}^{\text {square }}=\left\{\begin{array}{l}
-\mu_{c o}\left[\frac{4 J_{0}}{(2 n-1) \pi}+\frac{(2 n-1) \sigma_{c o} \omega}{r} C_{n}\right] \text { coil } \\
-\frac{(2 n-1) \sigma_{c r} \omega \mu_{c r}}{r} C_{n} \text { crucible } \\
0 \text { elsewhere }
\end{array}\right. \\
& \hat{\mathcal{L}} C_{n}^{\text {square }}=\left\{\begin{array}{l}
\frac{(2 n-1) \sigma_{c o} \omega \mu_{c o}}{r} S_{n} \text { coil } \\
\frac{(2 n-1) \sigma_{c r} \omega \mu_{c r}}{r} S_{n} \text { crucible } \\
0 \text { elsewhere }
\end{array}\right. \\
& q^{\text {square }}(r, z)=\sum_{n=1}^{\infty} q_{n}^{\text {square }}(r, z) \\
& =\left\{\begin{array}{l}
\sum_{n=1}^{\infty} \frac{(2 n-1)^{2} \sigma_{c o} \omega^{2}}{2 r^{2}}\left[S_{n}^{2}+\left(\frac{4 J_{0} r}{(2 n-1)^{2} \pi \sigma_{c o} \omega}+C_{n}\right)^{2}\right] \text { coil } \\
\sum_{n=1}^{\infty} \frac{(2 n-1)^{2} \sigma_{c r} \omega^{2}}{2 r^{2}}\left[S_{n}^{2}+C_{n}^{2}\right] \text { crucible }
\end{array}\right.
\end{aligned}
$$

\section{c) Triangle Waveform}

The triangle waveform of the input current in the coil is approximated as

$$
\begin{aligned}
& J_{d}=J_{0} F^{\text {triangle }}(t)=\frac{8 J_{0}}{\pi^{2}} \sum_{n=1}^{\infty} \frac{(-1)^{n-1}}{(2 n-1)^{2}} \sin (2 n-1) \omega t \\
& \psi_{B}^{\text {triangle }}=\sum_{n=1}^{\infty} \psi_{B(n)}^{\text {triangle }} \\
& =\sum_{n=1}^{\infty}\left[S_{n} \sin (2 n-1) \omega t+C_{n} \cos (2 n-1) \omega t\right]
\end{aligned}
$$

$$
\begin{aligned}
& J_{e}^{\text {triangle }}=\sum_{n=1}^{\infty} J_{e(n)}^{\text {triangle }} \\
& =\sum_{n=1}^{\infty} \frac{(2 n-1) \sigma \omega}{r}\left[C_{n} \sin (2 n-1) \omega t\right. \\
& -S_{n} \cos (2 n-1) \omega t \\
& \hat{\mathcal{L}} S_{n}^{\text {triangle }}=\left\{\begin{array}{l}
-\mu_{c o}\left[\frac{(-1)^{n-1} 8 J_{0}}{(2 n-1)^{2} \pi^{2}}+\frac{(2 n-1) \sigma_{c o} \omega}{r} C_{n}\right] \text { coil } \\
-\frac{(2 n-1) \sigma_{c r} \omega \mu_{c r}}{r} C_{n} \text { crucible } \\
0 \text { elsewhere }
\end{array}\right. \\
& \hat{\mathcal{L}} C_{n}^{\text {triangle }}=\left\{\begin{array}{l}
\frac{(2 n-1) \sigma_{c o} \omega \mu_{c o}}{r} S_{n} \text { coil } \\
\frac{(2 n-1) \sigma_{c r} \omega \mu_{c r}}{r} S_{n} \text { crucible } \\
0 \text { elsewhere }
\end{array}\right. \\
& q^{\text {triangle }}(r, z)=
\end{aligned}
$$

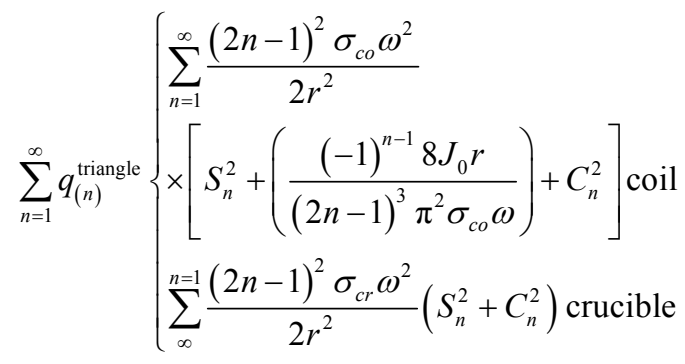

d) Sawtooth Waveform

The related equations of the sawtooth waveform of the input current can be written similar to the square and triangle waveforms. They are

$$
\begin{gathered}
J_{d}=J_{0} F^{\text {sawtooth }}(t)=-\frac{J_{0}}{\pi^{2}} \sum_{n=1}^{\infty} \frac{\sin n \omega t}{n} \\
\psi_{B}^{\text {sawtooth }}=\sum_{n=1}^{\infty} \psi_{B(n)}^{\text {sautooth }}=\sum_{n=1}^{\infty}\left[S_{n} \sin n \omega t+C_{n} \cos n \omega t\right]
\end{gathered}
$$

$J_{e}^{\text {sawtooth }}=\sum_{n=1}^{\infty} J_{e(n)}^{\text {sawtooth }}=\sum_{n=1}^{\infty} \frac{n \sigma \omega}{r}\left[S_{n} \sin n \omega t+C_{n} \cos n \omega t\right](26)$

$$
\hat{\mathcal{L}} S_{n}^{\text {sawtooth }}=\left\{\begin{array}{l}
-\mu_{c o}\left[\frac{-J_{0}}{n \pi}+\frac{n \sigma_{c o} \omega}{r} C_{n}\right] \text { coil } \\
-\frac{n \sigma_{c r} \omega \mu_{c r}}{r} C_{n} \text { crucible } \\
0 \text { elsewhere }
\end{array}\right.
$$




$$
\begin{gathered}
\hat{\mathcal{L}} C_{n}^{\text {sawtooth }}=\left\{\begin{array}{l}
\frac{n \sigma_{c o} \omega \mu_{c o}}{r} S_{n} \text { coil } \\
\frac{n \sigma_{c r} \omega \mu_{c r}}{r} S_{n} \text { crucible } \\
0 \text { elsewhere }
\end{array}\right. \\
q^{\text {sawtooth }}(r, z) \\
=\sum_{n=1}^{\infty} q_{(n)}^{\text {sawtooth }}\left\{\begin{array}{l}
\sum_{n=1}^{\infty} \frac{n^{2} \sigma_{c o} \omega^{2}}{2 r^{2}}\left[S_{n}^{2}+\left(\frac{-J_{0} r}{\pi n^{2} \sigma_{c o} \omega}\right)+C_{n}^{2}\right] \text { coil } \\
\sum_{n=1}^{\infty} \frac{n^{2} \sigma_{c r} \omega^{2}}{2 r^{2}}\left(S_{n}^{2}+C_{n}^{2}\right) \text { crucible }
\end{array}\right.
\end{gathered}
$$

\subsection{The Calculation Conditions}

The driving current density in the induction coil is calculated by $J_{0}=\sigma_{c o} V_{\text {coil }} /\left(2 \pi R_{\text {coil }} N\right)$, where $V_{\text {coil }}$ is the total voltage of the coil, $R_{\text {coil }}$ is the mean value of the coil radius and $N$ is the number of coil turns. The boundary conditions are $\psi_{B}=0$; both in the far field $(r, z \rightarrow \infty)$ and at the axis of symmetry $(r=0)$.

Values of electrical conductivity employed for our calculations are presented in [9], operating parameters are listed in Table $\mathbf{1}$ and the geometry of the growth heating system is shown in Figure 2. The fundamental partial equations require using a numerical discretization method to solve them. Calculation of the equations with boundary conditions has been made by $2 \mathrm{D}$ finite element method.

The two-dimensional computational domain with the finite element triangle mesh is shown in Figure 3. In the space close to and in the metal parts (i.e. crucible, afterheater and RF-coil) the mesh is denser because of the high gradients of the electromagnetic fields. After solving the set of equations, we can obtain the electromagnetic field structure in the system as well as the volumetric power distribution in the crucible, afterheater and RF-coil.

Table 1. Operating parameters used for calculations.

\begin{tabular}{ccc}
\hline Description (units) & Symbol & Value \\
\hline Crucible inner radius $(\mathrm{mm})$ & $r_{c}$ & 50 \\
Crucible wall thickness $(\mathrm{mm})$ & $l_{c}$ & 2 \\
Crucible inner height $(\mathrm{mm})$ & $h_{c}$ & 100 \\
Baffle inner radius $(\mathrm{mm})$ & $r_{b}$ & 35 \\
Bottom heater height $(\mathrm{mm})$ & $h_{b h}$ & 50 \\
Height of the thick bottom $(\mathrm{mm})$ & $h_{t b}$ & 10 \\
Radius of the round bottom corner $(\mathrm{mm})$ & $r_{c b}$ & 10 \\
Coil inner radius $(\mathrm{mm})$ & $r_{c o}$ & 78 \\
Coil width (mm) & $l_{c o}$ & 13 \\
Coil wall thickness $(\mathrm{mm})$ & $l_{c o}$ & 1.5 \\
Height of coil turns (mm) & $h_{c o}$ & 20 \\
Distance between coil turns $(\mathrm{mm})$ & $d_{c o}$ & 3 \\
Total voltage of the RF-coil $(v)$ & $V_{\text {coil }}$ & 200 \\
Current frequency of RF-coil $(\mathrm{kHz})$ & $f$ & 10 \\
\hline
\end{tabular}

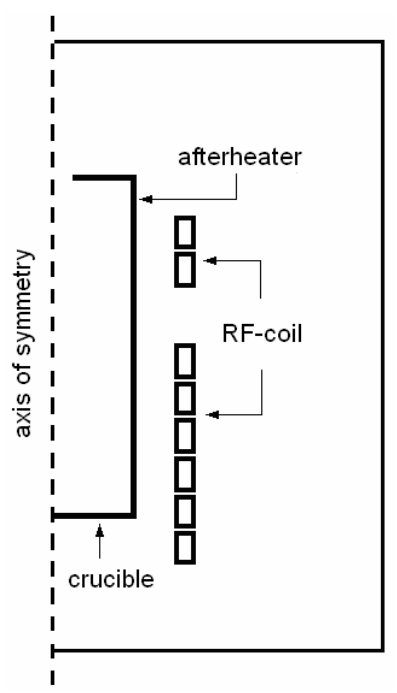

Figure 2. Sketch of an oxide Czochralski growth heating.

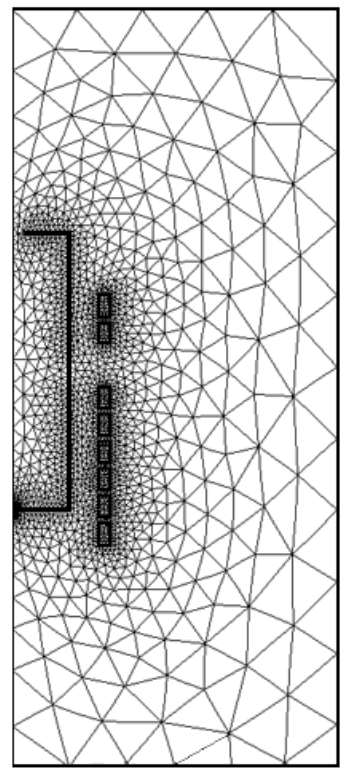

Figure 3. The finite element mesh structure of the calculation domain.

\section{Results and Discussion}

We explain the results of electromagnetic field and heating pattern in an oxide $\mathrm{CZ}$ setup including a cylindrical metal crucible, active afterheater and RF-coil corresponding to a real growth situation with different shapes of driving current in the RF-coil and with unique amplitude and frequency.

\subsection{Electromagnetic Fields}

Figures 4-7 show the distribution of in-phase component and out-of-phase component of the magnetic stream function $\left(\psi_{B}\right)$ for the cases of sine, square, triangle, and sawtooth waveforms, respectively, in the growth setup. 


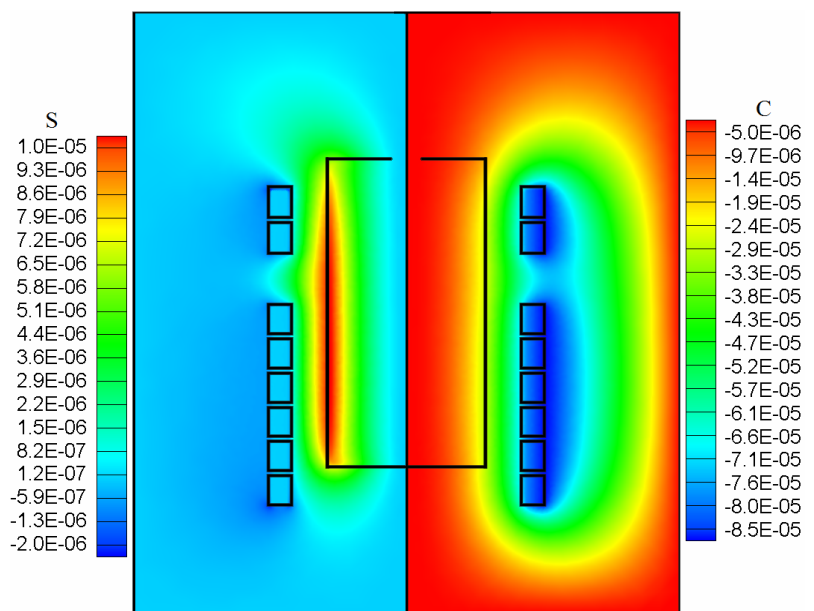

Figure 4. Components of the magnetic stream function $\left(\psi_{B}\right)$ calculated for the case of sine waveform. The left hand side shows the in-phase component $(S)$ and the right hand side shows the out-of-phase component $(C)$ in the setup.

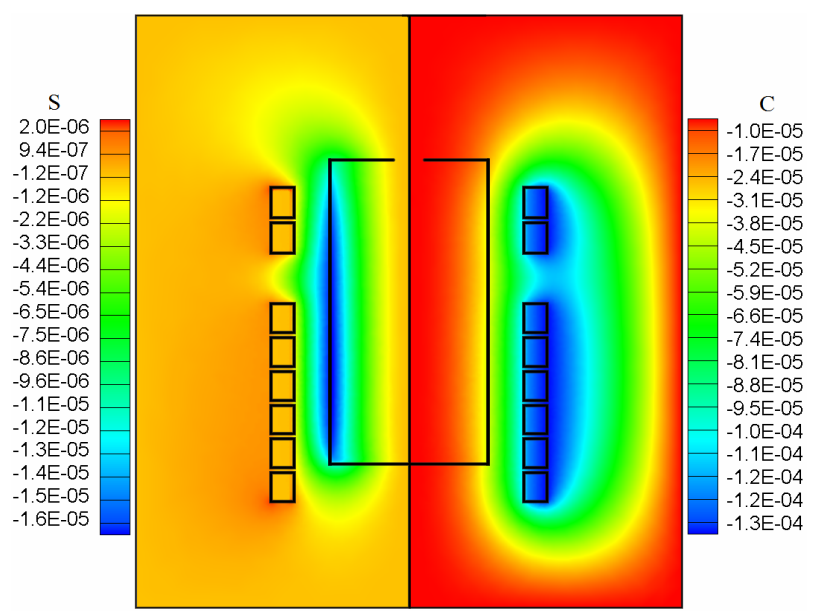

Figure 5. Components of the magnetic stream function $\left(\psi_{B}\right)$ calculated for the case of square waveform. The left hand side shows the in-phase component $(S)$ and the right hand side shows the out-of-phase component $(C)$ in the heating setup.

The maximum of in-phase component $\left(S_{\max }\right)$ is located at the lowest and top edges of the RF-coil while the minimum $\left(S_{\min }\right)$ is located on the middle of the outer surface of crucible and afterheater wall, for the square and triangle waveforms. But for the sine and sawtooth waveforms, it is vice versa, that is, the positions of the $\left(S_{\max }\right)$ and $\left(C_{\max }\right)$ are replaced. For the out-of-phase component $(C)$, the minimum is located on the outer surfaces of the induction coil turns for the cases of sine, square and triangle waveforms while the maximum is placed there for the case of sawtooth waveform. The distribution of $C$-component has a linear gradient in the space between the coil and the crucible and afterheater wall for all cases. The crucible and afterheater wall squeezes this compo-

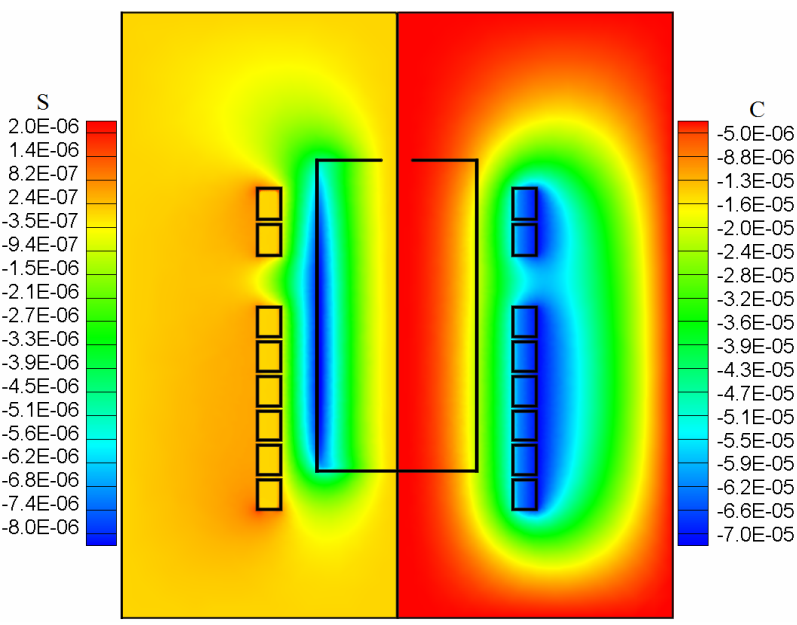

Figure 6. Components of the magnetic stream function $\left(\psi_{B}\right)$ calculated for the case of triangle waveform. The left hand side shows the in-phase component $(S)$ and the right hand side shows the out-of-phase component $(C)$ in the heating setup.

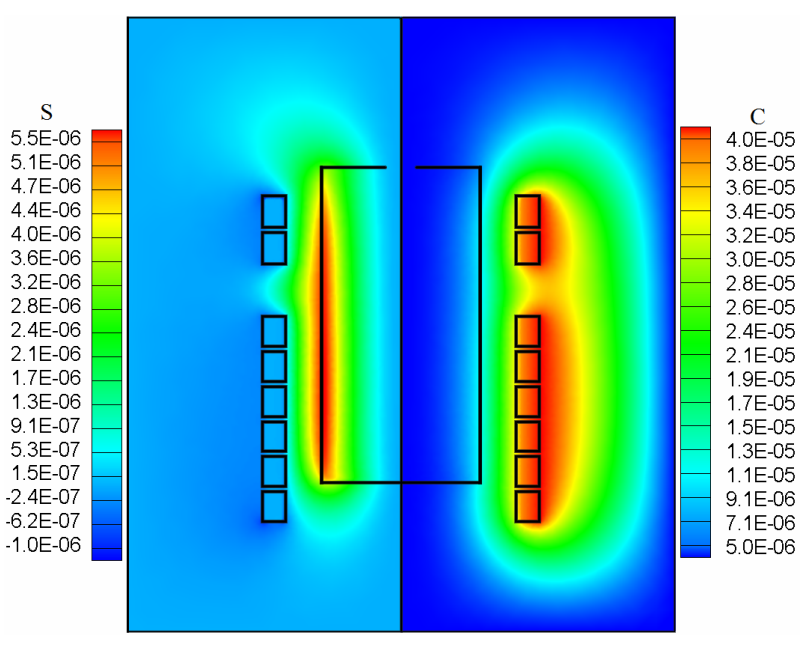

Figure 7. Components of the magnetic stream function $\left(\psi_{B}\right)$ calculated for the case of sawtooth waveform. The left hand side shows the in-phase component $(S)$ and the right hand side shows the out-of-phase component $(C)$ in the heating setup.

nent to the area between the crucible and afterheater, and the RF-coil. Some interesting advantages are:

- The gradient of the $S$-component is too high in the area close to the maximum and minimum points, which is not true for other parts of the system;

- Deformation and distortion of the $S$-component in the area close to the extreme edges of the RF-coil are particularly visible for all cases (edge effect). For the crucible and afterheater, spatial distribution of the $S$ component is along and parallel to their sidewall;

- The strongest electromagnetic fields belong to the square waveform while the weakest fields are produced by the sawtooth waveform. 


\subsection{Heat Generation}

The volumetric heat generation rate $(q)$ in the crucible and afterheater has been shown for all cases in Figure 8. The power intensity is at its maximum value at the middle portion of the outer surface of the crucible sidewall, which arises from the skin effect.

The most important features are:

- The heating structure of the crucible and afterheater is the same for all cases expect for their intensity. The most powerful energy is produced by square, sine, triangle and sawtooth waveform, respectively, Figure 9. This feature is predictable from the related electromagnetic fields distribution;

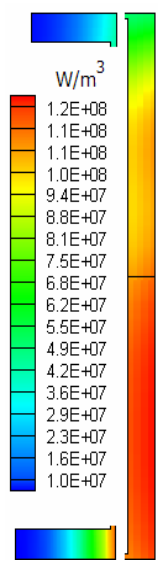

(a)

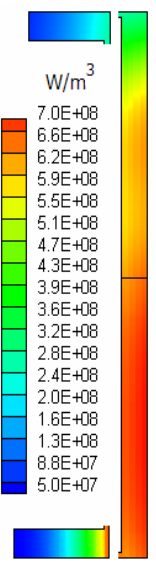

(b)

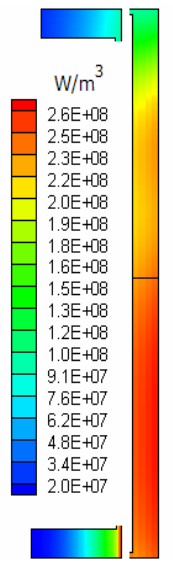

(c)

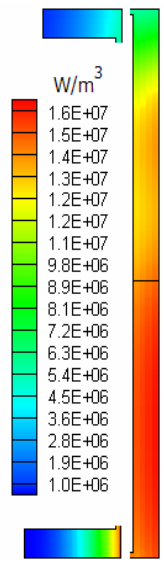

(d)
Figure 8. Volumetric power distribution $(q)$ in the crucible and afterheater computed for (a) sine; (b) square; (c) triangle; and (d) sawtooth waveform of the driving current (for a better demonstration the crucible and afterheater sidewall and bottom are separately magnified).

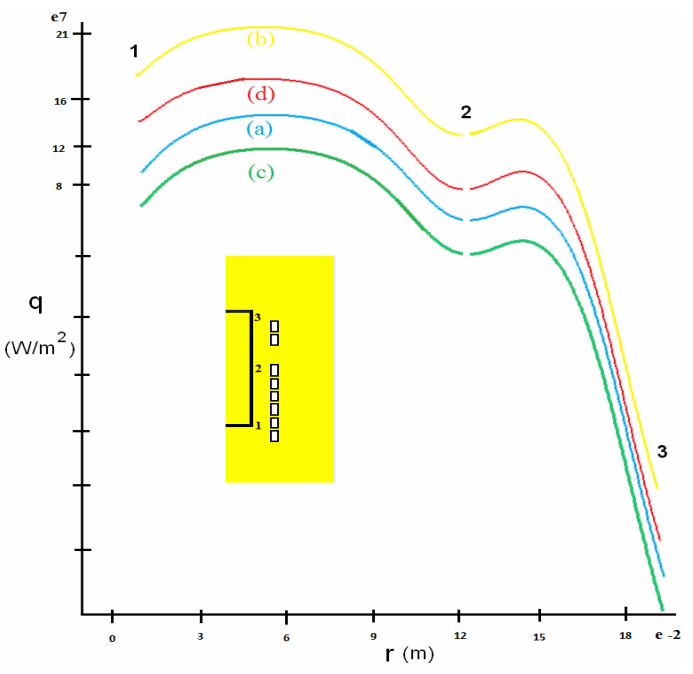

Figure 9. Profiles of the heat generated along the outer surface of the crucible and afterheater side wall calculated for (a) sine; (b) square; (c) triangle; and (d) sawtooth waveform of the input current.
- The spatial distribution of heat generation in the induction coil is mostly uniform with local "hot spots" (highly heated areas) at the lowest and upper edges, which is shown in Figure 10. The skin effect and proximity effect are responsible for appearance of these undesirable overheating because the induced eddy currents are concentrated on the top and lowest corners of the RF-coil [10,11];

- It is worth to note that despite of different total power generation, the coil efficiency (i.e., the part of the energy delivered to the coil that is transferred to the workpiece) does not change and is approximately the same for all cases (Table 2).

\section{Conclusions}

To study the dependence of electromagnetic distribution and heating pattern on the input current shape (sine, square, triangle and sawtooth waveforms) of the induction coil, a set of 2D numerical calculations was performed.
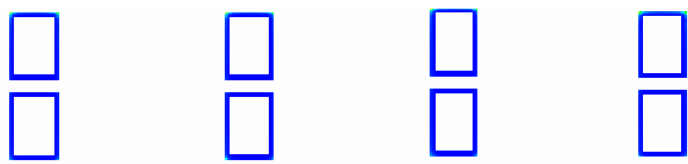

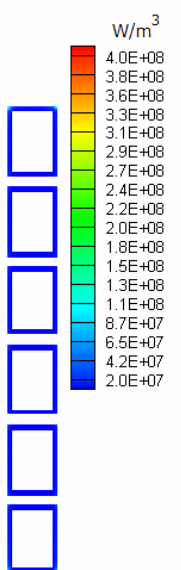

(a)

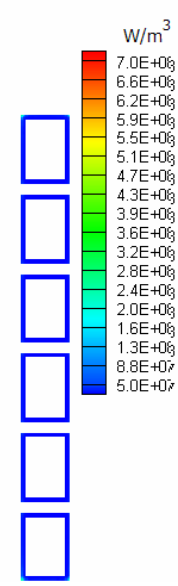

(b)

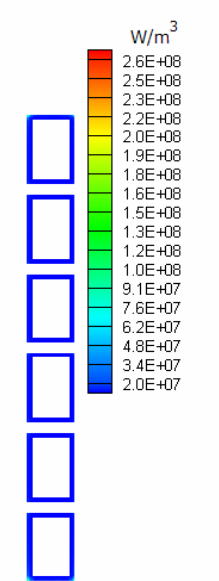

(c)

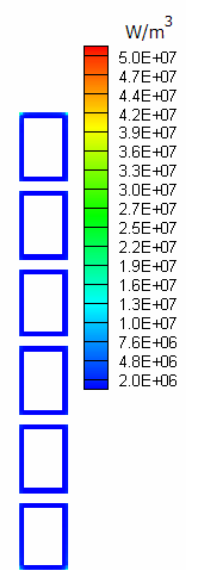

(d)
Figure 10. Volumetric power distribution $(q)$ in the induction coil calculated for (a) Sine; (b) Square; (c) Triangle; and (d) Sawtooth waveform of the input current.

Table 2. Detail information about the heat generated in the $\mathrm{CZ}$ coil (Heating efficiency is the part of the energy delivered to the coil that is transferred to the crucible and afterheater).

\begin{tabular}{cccc}
\hline Waveform & $\begin{array}{c}\text { Crucible and } \\
\text { afterheater }(\mathrm{kW})\end{array}$ & $\begin{array}{c}\text { Induction coil } \\
(\mathrm{kW})\end{array}$ & $\begin{array}{c}\text { Heating } \\
\text { efficiency }(\%)\end{array}$ \\
\hline Sine & 15 & 1.4 & 91.5 \\
Square & 26 & 2.4 & 91.6 \\
Triangle & 10 & 0.9 & 91.5 \\
Sawtooth & 1.9 & 0.2 & 92.1 \\
\hline
\end{tabular}


From the computational results described above, we can conclude:

- The spatial structure of electromagnetic fields and generated heat is a complex function of several parameters such as setup geometry and driving current shape;

- The electromagnetic fields distribution within the crucible and afterheater as well as the RF-coil is not uniform. This electromagnetic fields nonuniformity causes a nonuniform heating pattern in the crucible and afterheater, which in turn leads to a nonuniform temperature profile in the growth system.

A square input current results in a high intense heating of the crucible and afterheater while a sawtooth waveform leads to a low heating intensity in that part of the system. Different amount of produced energy in the setup is due to differences in the intensity and distribution of the electromagnetic fields. Understanding the physics of these properties is important during designing of an induction system for certain crystal growth applications.

\section{REFERENCES}

[1] J. J. Derby, L. J. Atherton and P. M. Gresho, "An Integrated Process Model for the Growth of Oxide Crystals by the Czochralski Method," Journal of Crystal Growth, Vol. 97, No. 3-4, 1989, pp. 792-826. doi:10.1016/0022-0248(89)90583-6

[2] D. T. J. Hurle, "Crystal Pulling from the Melt," SpringerVerlag, Berlin, Heidelberg, 1993. doi:10.1007/978-3-642-78208-4

[3] O. Klein and P. Philip, "Transient Numerical Investigation of Induction Heating during Sublimation Growth of
Silicon Carbide Single Crystals," Journal of Crystal Growth, Vol. 247, No. 1-2, 2003, pp. 219-235. doi:10.1016/S0022-0248(02)01903-6

[4] M. H. Tavakoli, F. Samavat and M. Babaiepour, "Influence of Active Afterheater on the Induction Heating Process in Oxide Czochralski Systems," Crystal Research and Technology, Vol. 43, No. 2, 2008, pp. 145-151. doi:10.1002/crat.200710993

[5] M. H. Tavakoli, A. Ojaghi, E. Mohammadi-Manesh and M. Mansour, "Influence of Coil Geometry on the Induction Heating Process in Crystal Growth Systems," Journal of Crystal Growth, Vol. 311, No. 6, 2009, pp. 15941599. doi:10.1016/j.jcrysgro.2009.01.092

[6] M. H. Tavakoli, E. Mohammadi-Manes and A. Ojaghi, "Influence of Crucible Geometry and Position on the Induction Heating Process in Crystal Growth Systems," Journal of Crystal Growth, Vol. 311, No. 17, 2009, pp. 42814288. doi:10.1016/j.jcrysgro.2009.07.013

[7] M. H. Tavakoli, H. Karbaschi, F. Samavat and E. Mohammadi-Manesh, "Numerical Study of Induction Heating in Melt Growth Systems-Frequency Selection," Journal of Crystal Growth, Vol. 312, No. 21, 2010, pp. 3198-3203. doi:10.1016/j.jcrysgro.2010.07.035

[8] G. H. Hardy and W. W. Rogosinski, "Fourier Series," Dover, 1999.

[9] M. H. Tavakoli, "Modeling of Induction Heating in Oxide Czochralski Systems Advantages and Problems," Crystal Growth Design, Vol. 8, No. 2, 2007, pp. 483-488. doi: $10.1021 / \operatorname{cg} 070378+$

[10] S. Zinn and S. L. Semiatin, "Elements of Induction Heating," ASM International, Cleveland, 1988.

[11] V. Rudnev, D. Loveles, R. Cook and M. Black, "Handbook of Induction Heating," CRC Press, New York, 2003. 